\title{
Uso de plantas medicinais por indivíduos de uma comunidade do Acre
}

\author{
Use of medicinal plants by individuals from a community in Acre \\ Uso de plantas medicinales por participantes en una comunidad de Acre
}

\author{
Ruth Silva Lima da Costa \\ ORCID: https://orcid.org/0000-0003-1890-086X \\ Centro Universitário Uninorte, Brasil \\ E-mail: ruttylyma@gmail.com \\ Vanessa Victoria Alnert Vieira Tavares \\ ORCID: https://orcid.org/0000-0002-1819-5171 \\ Centro Universitário Uninorte, Brasil \\ E-mail: vanessa.alnert@gmail.com \\ Eduardo Souza Dourado \\ ORCID: https://orcid.org/0000-0001-8219-8812 \\ Centro Universitário Uninorte, Brasil \\ E-mail: edusouzadourado@gmail.com \\ Vanessa Holanda de Souza Ribeiro da Costa \\ ORCID: https://orcid.org/0000-0002-6598-5408 \\ Centro Universitário Uninorte, Brasil \\ E-mail: vanessamed.if@gmail.com \\ Yara Costa Lameira \\ ORCID: https://orcid.org/0000-0002-3154-5914 \\ Centro Universitário Uninorte, Brasil \\ E-mail: yclameira@gmail.com \\ Priscila Santos Américo \\ ORCID: https://orcid.org/0000-0003-4759-0338 \\ Centro Universitário Uninorte, Brasil \\ E-mail: priamericosantos@gmail.com \\ Raquel Rodrigues dos Santos \\ ORCID: https://orcid.org/0000-0002-7356-5606 \\ Secretaria de Estado de Saúde do Acre, Brasil \\ E-mail: raquelrodriguesczs@gmail.com
}

\begin{abstract}
Resumo
O objetivo foi demonstar o uso de plantas medicinais por indivíduos de uma comunidade do Acre. Trata-se de um estudo descritivo e exploratório, de abordagem qualitativa, elaborado a partir de entrevistas realizadas junto a 21 indivíduos, de ambos os sexos, residentes nas adjacências de um bairro periférico do município de Rio Branco - Acre, em cuja localidade se encontra uma horta comunitária com o cultivo de plantas medicinais. A população participante do estudo, pertencia em sua maioria ao sexo feminino, dentro da faixa etária de 54 a 80 anos e faziam uso de plantas medicinais frequentemente, além de as indicar para familiares e amigos. A maioria aprendeu sobre o uso das plantas com as mães e as avós. Cerca de 32 espécies foram citadas como utilizadas para os mais variados tratamentos, sendo que a maioria utilizava as ervas em forma de chá. Eles expressaram que as práticas do uso de fitoterápicos podem colaborar com a melhora do estado de saúde de quem faz uso delas e que acreditavam no poder de cura das mesmas, pois tiveram experiências próprias a partir do seu uso. A população estudada, utiliza as plantas medicinais, embasadas apenas em seu conhecimento popular tradicional e na indicação de outros, o que evidencia a necessidade da realização de pesquisas para o melhor conhecimento e difusão de informações das propriedades terapêuticas e tóxicas afim de se evitar futuras complicações.
\end{abstract}

Palavras-chave: Plantas medicinais; Fitoterapia; Comunidade.

\begin{abstract}
The objective was to demonstrate the use of medicinal plants by individuals from a community in Acre. This is a descriptive and exploratory study, with a qualitative approach, based on interviews conducted with 21 individuals, of both sexes, living in the vicinity of a peripheral neighborhood in the city of Rio Branco - Acre, in whose locality they are located, a community garden with the cultivation of medicinal plants. The population participating in the study was mostly female, aged between 54 and 80 years old and used medicinal plants frequently, in addition to recommending them to family and friends. Most learned about the use plants from their mothers and grandmothers. About 32 species were cited as being used for the most varied treatments, most of which used herbs in the form of tea. They expressed that the practices of the use of herbal medicines can help improve the health status of those who use them and that they believed in their healing power, as they had their own experiences from their use. The population
\end{abstract}


studied uses medicinal plants, based only on their traditional popular knowledge and on the indication of others, which highlights the need to carry out research for better knowledge and dissemination of information on therapeutic and toxic properties in order to avoid future complications.

Keywords: Medicinal plants; Phytotherapy; Community.

\section{Resumen}

El objetivo era demostrar el uso de plantas medicinales por parte de personas de una comunidad de Acre. Se trata de un estudio descriptivo y exploratorio, con enfoque cualitativo, basado en entrevistas realizadas a 21 personas, de ambos sexos, residentes en las cercanías de un barrio periférico de la ciudad de Rio Branco - Acre, en cuya localidad se ubican huerto comunitario con cultivo de plantas medicinales. La población que participó en el estudio fue mayoritariamente femenina, con edades comprendidas entre los 54 y los 80 años y utilizaba plantas medicinales con frecuencia, además de recomendarlas a familiares y amigos. La mayoría aprendió sobre las plantas de sus madres y abuelas. Aproximadamente 32 especies fueron citadas como utilizadas para los tratamientos más variados, la mayoría de los cuales usaban hierbas en forma de té. Expresaron que las prácticas del uso de medicamentos a base de hierbas pueden ayudar a mejorar el estado de salud de quienes los usan y que creían en su poder curativo, ya que tenían sus propias experiencias con su uso. La población estudiada utiliza plantas medicinales, basándose únicamente en su conocimiento popular tradicional y en la indicación de otros, lo que pone de manifiesto la necesidad de realizar investigaciones para un mejor conocimiento y difusión de información sobre propiedades terapéuticas y tóxicas para evitar futuras complicaciones.

Palabras clave: Plantas medicinales; Fitoterapia; Comunidad.

\section{Introdução}

Desde os tempos mais remotos, observa-se o registro do uso terapêutico de plantas medicinais principalmente pelas civilizações da China, Índia, Egito e Grécia. E desde então, as pessoas vem utilizando-as com a finalidade de tratar e curar enfermidades, sendo esta utilização, relacionada com a própria evolução do homem, no qual mitos, lendas e tradiç̃̃es refletem o vasto emprego de plantas medicinais em todas as camadas sociais (Alves, 2013) .

A partir da segunda metade do século XX, com a grande evolução e avanço da medicina tradicional e o advento da indústria farmacêutica, a utilização ddo uso de plantas para tratamentos em saúde, ainda continua sendo realizada em larga escala, em especial por populações carentes, tendo em vista a dificuldade de acesso a centros de atendimentos hospitalares e a realização de exames, bem como a dificuldade de acesso à medicamentos. Esses motivos, aliados ao fácil acesso e à tradição do uso de plantas medicinais, têm contribuído para que essa terapêutica seja utilizada em larga escala por populações dos países em desenvolvimento ( Pinto et al., 2012).

Nas últimas duas décadas, alguns municípios brasileiros vêm incorporando Programas de Fitoterapia na atenção primária em saúde, com o objetivo de ampliar as opções terapêuticas e suprir carências medicamentosas de suas comunidades e, assim, melhorar a atenção à saúde ofertada aos usuários da rede pública (Ibiapiana, Leitão, Batista \& Pinto, 2014) , sendo assim, o Ministério da Saúde, em 2006, aprovou a Política Nacional de Práticas Integrativas e Complementares (PNPIC) no Sistema Único de Saúde (SUS), para garantir a integralidade na atenção à saúde, incentivando a prática do uso das plantas medicinais entre a população (Brasil, 2006).

Atualmente, a fitoterapia é considerada um assunto de interesse em saúde pública e portanto, cabe aos profissionais atuantes na Estratégia de Saúde da Família, esclarecerem dúvidas da população e realizarem orientações corretas quanto ao uso das plantas medicinais nas unidades de saúde e durante as visitas domiciliares, incentivando o seu uso de forma correta e apropriada (Arnous, Santos \& Beinner, 2005).

Dessa forma, muitos fatores têm contribuído para o aumento da utilização das plantas como recurso medicinal entre a população, dentre eles, o alto custo dos medicamentos industrializados, o difícil acesso da população à assistência médica, bem como a tendência ao uso de produtos de origem natural. Acredita-se que o cuidado realizado por meio da medicina natural, seja favorável à saúde humana, desde que o usuário tenha conhecimento prévio de sua finalidade, riscos e benefícios (Costa, Nogueira, Rafael, Rigotti \& Moura, 2013). 
Nesse sentido, torna-se relevante que os profissionais de saúde conheçam e discutam as práticas de saúde tradicionais e historicamente utilizadas em relação ao uso de plantas medicinais, a fim de orientar melhor sobre os riscos e beneficios da utilização de plantas medicinais, aliando o conhecimento popular ao científico. Dentro desse contexto, o presente estudo tem por objetivo analisar o uso de plantas medicinais em uma população de uma comunidade do Acre.

\section{Metodologia}

Trata-se de um estudo exploratório-descritivo de abordagem qualitativa, uma vez que esse tipo de estudo através de seus pressupostos metodológicos, é capaz de identificar um caminho natural para a compreensão de fenômenos comportamentais em determinado tempo, local e cultura. (Estrela, 2018; Pereira; Shitsuka; Parreira \& Shitsuka, 2018).

A população do estudo foi composta por 21 individuos, de ambos os sexos, residentes nas adjacências de um bairro periférico do município de Rio Branco - Acre, em cuja localidade se encontra uma horta comunitária com o cultivo de plantas medicinais.

Foram incluídos no estudo os moradores da região, que estivessem buscando atendimento na unidade de saúde da comunidade, de ambos os sexos, com idade igual ou superior a 50 anos e que não apresentavam transtorno ou doença mental ou em situação de substancial diminuição em sua capacidade de comunicação, além dos que concordaram em participar da pesquisa.

Foram excluídos os indivíduos, que frequentam a unidade, mas que não eram moradores da aréa de abrangência, que apresentavam transtornos ou doença mental ou em situação de substancial diminuição em sua capacidade de comunicação e os que se recusarem a participar do estudo.

A coleta de dados ocorreu através da aplicação de um formulário contendo 14 questões sobre o conhecimento e o uso de plantas medicinais no domicílio. Os participantes da pesquisa foram abordados na unidade de saúde durante a busca por atendimento.

O método de seleção se deu pela amostragem por conveniência. Após concordarem participar da pesquisa os mesmos foram esclarecidos através da leitura e assinatura do Termo de Consentimento Livre e Esclarecido.

A entrevista foi realizada em local calmo e tranquilo da unidade e teve a duração de até 20 minutos. Após a coleta de dados os dados, transcritos na íntegra em arquivo eletrônico. O tratamento dos dados foi realizado através da Análise Qualitativa de Conteúdo de Bardin, compondo-se de leituras flutuantes, emergindo núcleos de significados no conjunto do material sob análise (de Sousa \& dos Santos 2020).

A análise das entrevistas foi realizada pela categorização de dados: Primeiramente foi realizada a ordenação dos dados obtidos; seguido da sua classificação com a leitura exaustiva e repetida dos textos, estabelecendo interrogações para identificar o que surgiu de relevante. Por se tratar de uma pesquisa qualitativa, os dados foram analisados criteriosamente quanto as interligações de todas as questões do formulário de maneira individual e apresentados na forma de quadro e tabelas, com a descrição na íntegra das falas dos participantes referente as perguntas abertas.

Com o intuito de preservar a identidade das participantes do estudo, de acordo com a Resolução 466/12 que trata da pesquisa com seres humanos, será utilizada a letra $\mathrm{P}$ seguido de uma identificação numérica, nas citações das falas dos mesmos.

Esta pesquisa foi fundamentada nas recomendações da Resolução de $\mathrm{N}^{\circ}$ 466, de 12 de dezembro de 2012 do Conselho Nacional de Saúde que rege sobre a realização de pesquisa com informações sobre seres humanos, e foi aprovada no comitê de ética em pesquisa local através do parecer nº 4.137.971. 


\section{Resultados e Discussão}

Participaram da pesquisa 21 usuários, havendo um predomínio do gênero feminino. A faixa etária variou entre 54 e 80 anos. Quanto ao uso de plantas medicinais, todos relataram fazer uso pelo menos uma vez nos últimos 6 meses e indicado a sua utilização entre familiares e amigos. Mediante ao fato de quem os ensinou sobre a utilização das plantas para tratamentos em saúde, a maioria aprendeu com as mães e avós.

A utilização de plantas medicinais para fins terapêuticos é uma prática tradicional, muito utilizada entre a população, tendo especificidades em cada região, sendo o seu uso muito frequente, principalmente entre a população idosa (Araújo, 2018).

Os achados do presente estudo, vão de encontro com os resultados de uma pesquisa realizada na cidade de Quixadá $\mathrm{CE}$, onde a maioria dos entrevistados foram mulheres e o maior número de informações e conhecimentos sobre plantas medicinais concentrou-se na faixa de 51-60 anos e que faziam uso de plantas medicinais de forma contínua e diariamente (Oliveira \& Lucena, 2015).

A prática do uso de plantas medicinais para fins terapêuticos, vem sendo recomendada por organismos internacionais e também no Brasil através da política nacional de plantas medicinais e fitoterápicos, cujo objetivo é valorizar os saberes tradicionais no cuidado à saúde adquiridos ao longo dos séculos (Castro \& Figueiredo, 2019).

No que se refere ao conhecimento adquirido sobre as plantas medicinais e sua forma de utilização, os resultados deste estudo evidenciaram que a maioria aprendeu com as mães e avós, fato esse que vai de encontro aos achados da literatura, onde conclui-se o repasse do conhecimento empírico ao longo das gerações, principalmente entre membros da mesma família, bem como das indicações feitas por amigos (dos Santos, dos Santos, Magnata, Garcia \& Martins, 2016).

Sendo assim é relevante enfatizar que há a necessidade de se resgatar e valorizar o conhecimento popular sobre a utilização de plantas medicinais, ressaltando a sua forma correta de uso, devido ao fato de que com o passar do tempo, a população passou a ter mais condições de vida, o que levou a muitas vezes, deixar de lado a prática de uso de fitoterápicos, em detrimento de medicamentos industrializados, comprometendo-se assim, a transmissão dos saberes populares para as futuras gerações ( Silva et al., 2020). 
Quadro 1: Principais plantas medicinais utilizadas, indicações e formas de uso.

\begin{tabular}{|c|c|c|c|}
\hline Planta Medicinal & Nome Científico & Indicações & Forma de uso \\
\hline Alecrim & Rosmarinus officinalis $L$. & Calmante & Chá \\
\hline Alfavaca & Ocimum gratissimum $L$ & Infecção urinária & Chá \\
\hline Agrião & Nasturtium officinale & Bronquite & Chá \\
\hline Algodão Roxo & Gossypium herbaceum & Febre e Anti-inflamatório & Chá \\
\hline Boldo & Coleus barbatus $L$ & $\begin{array}{l}\text { Fígado inflamado, dor no } \\
\text { estômago, dor de garganta }\end{array}$ & Chá \\
\hline Capim Santo & Cymbopogon citratus & Calmante & Chá \\
\hline Capeba & Piper umbellatum $L$ & Inflamação de ouvido & Chá \\
\hline Casaca do Jatobá & Hymenaea courbaril L. & $\begin{array}{c}\text { Vitamina, tosse, anti- } \\
\text { inflamatório, antibiótico, gripe. }\end{array}$ & Chá, suco \\
\hline Carmelitana & Lippia Alba (Mill.) & Febre e dor de cabeça & Chá \\
\hline Cerca Canteiro & Schefflera arboricola & Dor no estômago & Chá \\
\hline Copaíba & Copaifera langsdorffii Desf. & $\begin{array}{c}\text { Fígado inflamado e garganta } \\
\text { inflamado }\end{array}$ & Chá \\
\hline Cidreira & Hedyosmum brasiliense Miq & Calmante & Chá \\
\hline Crajiru & Arrabidaea chica & Inflamação & Chá, emplasto \\
\hline Corama & Bryophyllum pinnata & Cicatrização & Chá, emplasto \\
\hline Eucalipto & Eucalyptus sp. & Gripe & Chá, lambedor \\
\hline Espinheira Santa & Maytenus ilicifolia & Gastrite & Chá \\
\hline Folha de Laranja & Citrus aurantium L & Dor no estômago & Chá \\
\hline Folha de Azeitona & Olea europaea L & Diarréia & Chá \\
\hline Gengibre & Zingiber officinale L & Gripe & Chá, mastigar \\
\hline Hortelã & Mentha sp & Gripe, calmante, insônia & Chá \\
\hline Jambu & Acmella oleracea & Gripe & Chá \\
\hline Malvarisco & $\begin{array}{c}\text { Plectranthus amboinicus } \\
\text { (Lour.) }\end{array}$ & Gripe & Chá \\
\hline Mastruz & Chenopodium ambrosioides $L$ & Gripe, Tosse, imunidade & Chá, lambedor \\
\hline Noni & Morinda citrifolia $L$ & Inflamação da próstata & Chá \\
\hline Óleo de Andiroba & Carapa guianensis Aubl & Diabetes & Tomar puro \\
\hline Óleo de Pequi & Caryocar brasiliense & Antibiótico & Tomar puro \\
\hline Podarco Roxo & Handroanthus impetiginosus & Infecções e inflamações & Chá \\
\hline Pacova & Philodendron martiaum & Dor no Infecções e inflamações & Chá \\
\hline Quina Quina & Cinchona spp & $\begin{array}{c}\text { Para azia, evitar o coronavírus, } \\
\text { malária }\end{array}$ & Chá \\
\hline Romã & Punica granatum L. & $\begin{array}{c}\text { Gripe, azia, melhorar a } \\
\text { imunidade }\end{array}$ & $\begin{array}{l}\text { Chá, mastigar a } \\
\text { casca }\end{array}$ \\
\hline Súcuuba & Himatanthus sucuuba) & Rins, coluna, fígado e diarreia & Chá \\
\hline Unha de gato & Uncaria tomentosa & Inflamação na próstata & Chá \\
\hline
\end{tabular}

Fonte: Autores (2021).

A partir da análise do Quadro 1, observa-se que 32 espécies de plantas medicinais foram citadas como utilizadas entre os participantes do estudo, para os mais variados tipos de tratamento em saúde, sendo que a maioria fazia uso das ervas em forma de chá.

Mediante a esses achados, eles corroboram com um estudo realizado com idosos que frequentavam uma unidade de atenção primária em saúde, onde a maior parte deles fazia uso de plantas como tratamento de saúde e entre as mais citadas destacaram-se a hortelã, cidreira, boldo e alecrim, sendo a principal forma de preparo a infusão, utilizada três vezes na semana. Quanto ao motivo de uso, eles achavam melhor para curar, por não fazer mal a saúde e por gostarem mais do que o medicamento da farmácia (Szerwiesk, Cortez, Bennemann, Silva \& Cortez, 2017).

Um outro estudo realizado na cidade de Palotina - PR, detectou que a maioria dos participantes faziam uso de plantas medicinais, por ser um produto natural, cujo conhecimento para o uso, foi obtido através da tradição familiar, sendo as ervas cidreira, hortelã, boldo, camomila, guaco e a marcela as plantas mais utilizadas, e o resfriado, a gripe, cólicas e a enxaqueca 
como a doença e/ou mal-estar mais frequentemente tratados com elas (Stefanello et al, 2018).

Ainda com relação a utilização de plantas medicinais pela população, os achados de uma pesquisa realizada em Goiás em um centro de educação infantil, evidenciaram o uso frequente de chás de hortelã, boldo, camomila e erva-cidreira para tratamento das crianças, sendo que a alternativa da utilização de fitoterápicos segundo as mães, seria uma possibilidade acessível e eficiente (Motta, Lima \& Vale, 2016).

Evidencia-se que prática da utilização de plantas medicinais para fins terapêuticos é muito frequente entre a população. Um estudo etnobotânico realizado afim de medir o conhecimento e uso das plantas medicinais no município de Buriticupu - MA, demostrou que as espécies hortelã, capim-santo, erva-cidreira, malva-do-reino e folha santa, as mais frequentemente utilizadas, sendo as folhas, a parte botânica mais usada para tratamento de doenças infecciosas e parasitárias, doenças do sistema digestivo e sistema reprodutor feminino (de Melo Alencar et al., 2019).

Destarte, quando os participantes do presente estudo foram indagados sobre de que modo achavam que as plantas medicinais contribuíam para a recuperação da saúde das pessoas, eles verbalizaram que acreditavam no poder de cura das plantas, pois as mesmas poderiam colaborar com a melhora do estado de saúde de quem faz uso delas, conforme falas expressas na Tabela 1.

Tabela 1: Conhecimento sobre a eficácia das plantas medicinais.

\begin{tabular}{cc}
\hline Participante & Resposta \\
\hline P1 & "Melhora a saúde das pessoas e não agride o organismo" \\
P2 & "Muito bom para a saúde" \\
P3 & "O efeito é mais lento, mais cura e não agride o organismo". \\
P4 & "Porque a gente melhora" \\
P5 & "Ajuda muito a gente na hora da doença e é de graça" \\
P6 & "Quem conhece sabe que é muito bom para saúde, melhor do que remédio de farmácia" \\
P7 & "Porque faz efeito mesmo" \\
P8 & "Serve para muita coisa" \\
P9 & "Porque é remédio natural que não tem efeitos colaterais". \\
P10 & "Porque cura as doenças mais comuns" \\
\hline
\end{tabular}

Fonte: Autores (2021).

Evidenciam-se através de estudos que os adeptos da utilização das plantas medicinais acreditam no seu poder de cura. O uso de fitoterápicos é favorável à saúde humana, desde que o usuário tenha conhecimento prévio de sua finalidade, riscos e benefícios, nesse sentido os profissionais de saúde, devem orientar a população quanto à utilização segura e racional do seu uso (de Fátima Colet et al., 2015).

Nesse sentido, o uso de fitoterápicos pela população, tem levantado o interesse de profissionais de saúde, afim de entender melhor quais as plantas mais utilizadas, indicações de uso, formas de preparo, detectar as crenças sobre seu efeito e a extensão de sua indicação e assim poder orientar a população sobre plantas medicinais que por ventura possam ter efeito nocivo sobre a saúde delas e assim desaconselhar o uso, evitando maiores complicações (dos Santos, da Silva Alves, Barros \& Pessoa, 2019).

Sendo assim, estudos vêm evidenciando o uso de plantas medicinais pelos usuários do Sistema Único de Saúde (SUS), sendo que essa forma terapêutica apesar de menos utilizadas do que a medicina convencional, continuam sendo uma alternativa terapêutica para parte da população, principalmente a feminina, com mais idade e que frequentam as unidades de 
atenção primária em saúde (Zeni, Parisolto, Mattos \& Helena, 2017).

Frente ao questionamento quanto ao porquê os participantes do estudo creditavam no poder das plantas medicinais, a maioria verbalizou que reconheciam que elas podiam curar doenças, pois tiveram experiências próprias a partir do uso. Eis as falas expostas na Tabela 2.

Tabela 2: Motivos que os levaram a acreditar no poder de cura das plantas medicinais.

\begin{tabular}{cl}
\hline Participante & Resposta \\
\hline P11 & "Porque são da natureza e são puras" \\
$\mathbf{P 1 3}$ & "Sim porque já tomei muito e fez efeito sem precisar do remédio de farmácia" \\
$\mathbf{P 1 4}$ & "Porque é natural e a gente se sente bem tomando". \\
$\mathbf{P 1 5}$ & "Porque são da natureza e foi Deus que deixou para gente usar" \\
$\mathbf{P 1 6}$ & "Porque vem de muito tempo e na fé a gente vai usando e acreditando que dá certo" \\
$\mathbf{P 1 7}$ & "Porque regenera o corpo da gente" \\
$\mathbf{P 1 8}$ & "Porque meu irmão tomou e ficou curado do pâncreas" \\
$\mathbf{P 1 9}$ & "Melhora, mais curar acho que não, só remédio de farmácia" \\
$\mathbf{P 2 0}$ & "Sim, tomando com fé cura mesmo". \\
$\mathbf{P 2 1}$ & "Porque desde o começo do mundo as pessoas usam e dá certo" \\
\hline
\end{tabular}

Fonte: Autores (2021).

Entende-se que o uso das plantas medicinais, exerce um papel importante de fé no sistema de cuidado familiar, além da relação biológica do cuidado em saúde, onde os adeptos dessa prática, acreditam que estão fazendo o bem a si mesmos e aos outros através da utilização e/ou indicação dos fitoterápicos (Lima, Lima, Mendonça\& Lopes, 2017).

No entanto, estudo evidenciam que o cuidado de saúde com produtos à base de plantas medicinais é sim benéfico e favorável à saúde humana, desde que o usuário tenha conhecimento sobre sua finalidade, riscos e benefícios (Iserhard, Budó, Neves e Badke, 2009).

Sendo assim, torna-se importante conhecer o perfil de uso de plantas pela população, qualificando o conhecimento da mesma sobre estas terapias, relacionando o conhecimento popular aos estudos científicos, visando estabelecer critérios para o seu uso seguro (Gonçalves, Generutti. Chaves, Duarte \& Vila, 2011).

\section{Considerações Finais}

A população participante do estudo, pertencia em sua maioria ao sexo feminino, dentro da faixa etária de 54 a 80 anos e utilizava plantas medicinais frequentemente, além de as indicar para familiares e amigos, o que contribui para o desenvolvimento da medicina popular na comunidade.

A maioria aprendeu sobre o poder das plantas medicinais e suas indicações de uso com as mães e avós e cerca de 32 espécies de plantas foram citadas como utilizadas para os mais variados tipos de tratamento, sendo que a maioria as utilizava em forma de chás.

Eles expressaram acreditar, que a prática do uso de fitoterápicos, podem colaborar com a melhora do estado de saúde de quem faz uso delas e que acreditavam no poder de cura das mesmas pois tiveram experiências próprias a partir do uso.

No entanto, a utilização das plantas medicinais, não deve ser embasada apenas no conhecimento popular, o que evidencia a necessidade e importância da realização de pesquisas com base etnobotânica para o melhor conhecimento das 
propriedades terapêuticas e toxicidade dessas plantas, afim de evitar riscos à saúde de quem faz uso delas.

Deste modo, para trabalhos futuros, sugere se a realização de análises fitoquímicas em partes das plantas mais frequentemente utilizadas pela população, afim de se comprovar a eficácia e a segurança desses produtos naturais.

\section{Referências}

Alves, L. F. (2013). Produção de fitoterápicos no Brasil: história, problemas e perspectivas. Revista Virtual de Química, 5(3), 450-513.

Araújo, K. A. (2018). Conhecimento local e o uso de plantas medicinais em Boa Vista/Roraima-novas estratégias em saúde coletiva.

Arnous, A. H., Santos, A. S., \& Beinner, R. P. C. (2005). Plantas medicinais de uso caseiro-conhecimento popular e interesse por cultivo comunitário. Revista espaço para a saúde, 6(2), 1-6.

Brasil. Ministério da Saúde. Secretaria de Atenção à Saúde. Departamento de Atenção Básica. Política Nacional de Práticas Integrativas e Complementares no SUS - PNPIC-SUS. 2006. 92p. https://aps.saude.gov.br/politicas/pnpic.

Castro, M. R., \& Figueiredo, F. F. (2019). Saberes tradicionais, biodiversidade, práticas integrativas e complementares: o uso de plantas medicinais no SUS. Hygeia-Revista Brasileira de Geografia Médica e da Saúde, 15(31), 56-70

Costa, E. M., Nogueira, F., Rafael, E. T., Rigotti, T. R., \& Moura, S. E. (2013). Saberes e práticas populares de cuidado em saúde com o uso de plantas medicinais. In Saberes e práticas populares de cuidado em saúde com o uso de plantas medicinais (pp. 1-1).

de Fátima Colet, C., Cavalheiro, C. A. N., Dal Molin, G. T., Cavinatto, A. W., Schiavo, M., Schwambach, K. H., \& Oliveira, K. R. (2015). Uso de plantas medicinais por usuários do serviço público de saúde do município de Ijuí/RS. Revista brasileira de medicina de família e comunidade, 10(36).

de Melo Alencar, E., Cajaiba, R. L., Martins, J. D. S. C., Cordeiro, R. S., de Sousa, E. S., \& de Almeida Sousa, V. (2019). Estudo etnobotânico do conhecimento e uso das plantas medicinais no município de Buriticupu, Maranhão, Brasil. Revista Ibero-Americana de Ciências Ambientais, 10(6), 328-338.

de Sousa, J. R., \& dos Santos, S. C. M. (2020). Análise de conteúdo em pesquisa qualitativa: modo de pensar e de fazer. Pesquisa e Debate em Educação, 10(2), 1396-1416.

dos Santos, J. A. A., dos Santos, E. C. B., Magnata, S. D. S. L. P., Garcia, J. E., \& Martins, R. D. (2016). Diagnóstico e educação em saúde no uso de plantas medicinais: relato de experiência. Revista Ciência em Extensão, 12(4), 183-196.

dos Santos, S. L. F., da Silva Alves, H. H., Barros, K. B. N. T., \& Pessoa, C. V. (2019). Uso de Plantas Medicinais por idosos de uma instituição filantrópica. Revista Brasileira de Pesquisa em Ciências da Saúde, 4(2), 71-75.

Estrela, C. (2018). Metodologia científica: ciência, ensino, pesquisa. Artes Médicas.

Gonçalves, N. M. T., Generutti, M., Chaves, D. A. S., Duarte, M. M., \& Vila, C. (2011). A tradição popular como ferramenta para a implantação da fitoterapia no município de Volta Redonda-RJ. Rev Bras Farm, 92(4), 346-51.

Ibiapina, W. V., Leitão, B. P., Batista, M. M., \& Pinto, D. S. (2014). Inserção da fitoterapia na atenção primária aos usuários do SUS. Revista de Ciências da Saúde Nova Esperança, 12(1), 60-70.

Iserhard, A. R. M., Budó, M. D. L. D., Neves, E. T., \& Badke, M. R. (2009). Práticas culturais de cuidados de mulheres mães de recém-nascidos de risco do sul do Brasil. Escola Anna Nery, 13, 116-122.

Lima, C. A. B. D., Lima, Â. R. A., Mendonça, C. V., Lopes, C. V., \& Heck, R. M. (2017). O uso das plantas medicinais e o papel da fé no cuidado familiar. Revista Gaúcha de Enfermagem, 37.

Motta, A. O., Lima, D. C. S., \& Vale, C. R. (2016). Levantamento do uso de Plantas Medicinais em um Centro de Educação Infantil em Goiânia-GO. Revista da Universidade Vale do Rio Verde, 14(1), 629-646.

Oliveira, D. S., \& Lucena, E. M. P. (2015). O uso de plantas medicinais por moradores de Quixadá-Ceará. Revista Brasileira de Plantas Medicinais, 17, 407412.

Pereira, A. S., Shitsuka, D. M., Parreira, F. J., \& Shitsuka, R. (2018). Metodologia da pesquisa científica.

Pinto, D. S., de Araújo, I. M., dos Santos Oliveira, F. B., de Brito, F. R., de Menezes, M. A. M., \& Barbosa, W. A. S. (2012). Vivência de Acadêmicos de Saúde na Prática da Fitoterapia em Três Comunidades da Cidade de João Pessoa-Paraíba. Revista de Ciências da Saúde Nova Esperança, 10(2), 112-120.

Silva, F. N. S., Silva, R. G., Barbosa, I. J., Freitas, V. L. B., de Oliveira Firmino, D., \& Zuliani, D. Q. (2020). Valorização do conhecimento popular sobre o uso de plantas medicinais na terceira idade. Cadernos de Agroecologia, 15(2).

Stefanello, S., Kozera, C., Ruppelt, B. M., Fumagalli, D., Camargo, M. P., \& Sponchiado, D. (2018). Levantamento do uso de plantas medicinais na Universidade Federal do Paraná, Palotina-PR, Brasil. Extensão em Foco, 1(15).

Szerwieski, L. L. D., Cortez, D. A. G., Bennemann, R. M., Silva, E. S., \& Cortez, L. E. R. (2017). Uso de plantas medicinais por idosos da atenção primária. Revista eletrônica de enfermagem, 19.

Zeni, A. L. B., Parisotto, A. V., Mattos, G., \& Helena, E. T. D. S. (2017). Utilização de plantas medicinais como remédio caseiro na Atenção Primária em Blumenau, Santa Catarina, Brasil. Ciência \& Saúde Coletiva, 22, 2703-2712. 